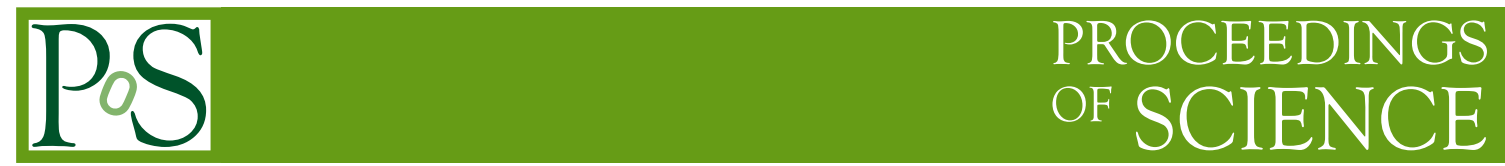

\title{
A new road to massive gravity?
}

\section{Eric Bergshoeff*, Marija Kovačević, Lorena Parra and Thomas Zojer}

Centre for Theoretical Physics, University of Groningen, 9747 AG Groningen, The Netherlands

E-mail: e.a.bergshoefferug.nl, m.kovacevicarug.nl,

l.parra.rodriguez@rug.nl, t.zojererug.nl

\begin{abstract}
We shortly review and discuss different ways of giving mass to the graviton. After discussing the linearized Fierz-Pauli theory we investigate two recent models of gravity in three space-time dimensions which both describe propagating massive spin-2 modes at the non-linear level. We comment on the particular advantages and shortcomings of these models with respect to each other. We conclude with possible generalizations of the two models.
\end{abstract}

Proceedings of the Corfu Summer Institute 2012

September 8-27, 2012

Corfu, Greece

${ }^{*}$ Speaker. 


\section{Motivation}

Einstein's theory of General Relativity (GR) is a very successful example of contemporary theoretical physics. It explains, and predicts, with remarkable accuracy, observables such as the perihelion shift of Mercury or the angle at which light is bent when passing by a very massive object; and in the low energy regime it perfectly agrees with classical Newtonian dynamics.

However, despite these successes there are problems at both ends of the energy scale. In the infrared regime we have difficulties matching observed galaxy rotation curves to theoretical models, forcing us to introduce the concept of dark matter. Furthermore, there is the infamous cosmological constant problem: the measured value of the cosmological constant is many orders of magnitude smaller than what we would naively expect from theoretical considerations. In the ultraviolet regime we would like to understand the singularities that plague our classical equations, by quantum effects of a putative quantum theory of gravity. Yet, there is no unique candidate for a quantum theory of gravity, one that would explain for example, microscopically, the entropy of black holes or the information loss paradox. ${ }^{1}$

These considerations motivate us to search for modifications of Einstein's theory of General Relativity. An overview of the vast variety of modifications to gravity that have been considered so far can be found in [1]. One idea, which could improve on both problems mentioned in the previous paragraph, and which will be the focus of our attention in this note, is to give a mass to the graviton. Long range interactions would be damped exponentially and this could narrow the gap between the expected and the observed value of the cosmological constant. Giving a mass to the graviton could also be relevant to the quantum aspects of the theory since, if the mass is introduced by higher-derivative terms in the action, it affects the (perturbative) renormalizability of the theory.

Thus, we will investigate how we can add a mass to the graviton, see also [2] for a review on massive gravity in particular. For concreteness, and not last for simplicity, we will focus on gravity in three space-time dimensions. In section 2 we start with a discussion of the linearized theory for a massive graviton, which was introduced by Fierz and Pauli. Then, in sections 3 and 4 respectively, we discuss two models of gravity that at the linearized level reduce to the Fierz-Pauli (FP) theory. Section 5 presents extensions and generalizations of the models described in sections 3 and 4. Finally, we conclude in section 6 and give an outlook on possible new roads to massive gravity and its extensions.

\section{Linearized massive gravity: Fierz-Pauli theory}

The question whether the graviton can have a (small) mass rather than being massless, as classical GR predicts, has been addressed already in 1939. Fierz and Pauli first proposed to modify GR at the linearized level by adding an explicit mass term to the Einstein-Hilbert action.

To describe the graviton, a spin-2 particle, one can use the standard representation of a rank-2 tensor $h_{\mu v}$, symmetric in its indices. In $d$ space-time dimensions it has $d(d+1) / 2$ independent components but not all of them describe spin-2 helicities. Some of these components describe spin-1 and spin-0 helicities. In order not to have any ghost degrees of freedom (DoF) the equation

\footnotetext{
${ }^{1}$ Though the gauge/gravity or AdS/CFT correspondence has enabled us to understand these phenomena much better.
} 
of motion of the massive field $h_{\mu v}$ should describe $(d-2)(d+1) / 2$ DoF. A massive graviton excitation in 3 dimensions has 2 DoF.

The starting point for the Fierz-Pauli (FP) equations is the Klein-Gordon equation for a massive spin-2 field $h_{\mu v}$. In order to describe the correct number of DoF we have to impose some constraints that will excise the unwanted DoF. To keep the total energy of the field positive and to avoid the helicities that do not correspond to spin-2 modes Fierz and Pauli additionally imposed a divergenceless condition, $\partial^{\mu} h_{\mu v}=0$, and a tracelessness condition, $\eta^{\mu v} h_{\mu v}=0$, on the field $h_{\mu \nu}$. Hence, the FP equations consist of a Klein-Gordon equation for a symmetric tensor field $h_{\mu \nu}$, together with a differential and an algebraic subsidiary constraint:

$$
\begin{aligned}
\left(\square-m^{2}\right) h_{\mu v} & =0, \\
\partial^{\mu} h_{\mu v} & =0, \\
h \equiv \eta^{\mu v} h_{\mu v} & =0 .
\end{aligned}
$$

This set of equations can be integrated to the FP Lagrangian

$$
\mathscr{L}_{\mathrm{FP}}=\frac{1}{2}\left\{h^{\mu v} G(h)_{\mu \nu}^{l i n}-m^{2}\left(h^{\mu v} h_{\mu v}-h^{2}\right)\right\},
$$

where $G(h)_{\mu \nu}^{\text {lin }}$ denotes the linearized Einstein tensor which has the following form in three dimensions:

$$
G(h)_{\mu \nu}^{\operatorname{lin}}=\varepsilon_{\mu}{ }^{\alpha \beta} \varepsilon_{v}^{\gamma \delta} \partial_{\alpha} \partial_{\gamma} h_{\beta \delta} .
$$

Note that the trace of the field $h_{\mu \nu}$ is included into the action although we require it to vanish onshell. In order to derive that all rank-1 $\left(\partial^{\mu} h_{\mu \nu}\right)$ modes vanish, it is necessary to show first that all rank-0 $\left(\partial^{\mu} \partial^{v} h_{\mu \nu}\right)$ modes vanish. One cannot obtain the latter constraint without introducing an additional scalar field $h$.

It is not possible to use a symmetric traceless tensor, $H_{\mu \nu}$, in order to construct an action that yields the FP equations. The reason for this is that one cannot construct equations of motion that have the same symmetry properties as the field itself. Consider for instance the equations

$$
\left(\square-m^{2}\right) H_{\mu v}=0, \quad \partial^{\mu} H_{\mu v}=0
$$

and rewrite them as

$$
\partial^{\alpha}\left(\partial_{\alpha} H_{\mu v}-\partial_{\mu} H_{\alpha v}\right)-m^{2} H_{\mu v}=0 .
$$

These equations are not symmetric and traceless, like $H_{\mu v}$, and therefore can not serve as the equations of motion for $H_{\mu \nu}$. If we were to start from the most general second-order derivative equations that are symmetric and traceless we find that we would always have to solve the constraint $\partial^{\mu} \partial^{v} H_{\mu \nu}=0$ first, in order to derive the subsidiary constraint $\partial^{\mu} H_{\mu v}=0$. Therefore, we would have to introduce an auxiliary field that will impose $\partial^{\mu} \partial^{v} H_{\mu \nu}=0$. For the FP theory, see eqs. (2.1) and (2.2), this additional field is the scalar $h$.

The special tuning of the mass term in the FP action is essential in order not to have any ghostlike DoF. Following [2] it is easy to show that the action (2.2) propagates only a massive spin-2 
mode, i.e. that there are not more than two DoF. For this purpose, we Legendre-transform the action (2.2) with respect to the spatial components. This leads to the following expressions for the spatial momenta ${ }^{2}$

$$
\pi_{i j}=\frac{\partial \mathscr{L}_{\mathrm{FP}}}{\partial \dot{h}_{i j}}=\dot{h}_{i j}-\dot{h}_{k k} \delta_{i j}-2 \partial_{(i} h_{j) 0}+2 \partial_{k} h_{0 k} \delta_{i j}
$$

and inverting for the velocities $\dot{h}_{i j}$ we obtain

$$
S_{\mathrm{FP}}=\int \mathrm{d}^{3} x\left\{\pi_{i j} \dot{h}_{i j}-\mathscr{H}+2 h_{0 i} \partial_{k} \pi_{k i}+m^{2} h_{0 k}^{2}+h_{00}\left(\nabla^{2} h_{k k}-\partial_{i} \partial_{j} h_{i j}-m^{2} h_{k k}\right)\right\} .
$$

The terms in $\mathscr{H}$ do not contain $h_{0 i}$ or $h_{00}$, nor any time derivatives. Thus, we note from (2.7) that $h_{00}$ appears as a Lagrange multiplier, independently of the value of $m^{2}$. This is due to the special tuning of the mass term in the FP Lagrangian (2.2). If the relative coefficient of the two contributions to the mass term was not exactly $-1, h_{00}$ would appear quadratically in (2.7). For a generic $m$ we have three DoF for the symmetric tensor $h_{i j}$, minus one constraint coming from $h_{00}$, giving two DoF for a massive spin-2 in three dimensions. If $m=0$, then the $h_{0 i}$ become also Lagrange multipliers, and we have to subtract another two DoF leaving no DoF for the massless spin-2.

The kinetic term of the FP action stems from the Einstein term and thus, being invariant under diffeomorphisms, has the gauge symmetry

$$
\delta h_{\mu v}=\partial_{\mu} \zeta_{v}+\partial_{v} \zeta_{\mu}
$$

Certainly, the mass term in (2.2) breaks the diffeomorphism invariance of the theory. Using Stückelberg fields one could by hand reintroduce such an invariance at the cost of introducing additional gauge fields.

It is known that the FP theory suffers from the van Dam-Veltman-Zakharov (vDVZ) discontinuity [3]. Namely, the massless limit of the FP theory does not give just linearized General Relativiy. This is due to the fact that the scalar mode does not decouple. This can be verified using the Stïckelberg fields mentioned above and coupling the massive field $h_{\mu v}$ to matter via a conserved energy-momentum tensor $T_{\mu \nu}$. Then, in the massless limit one observes a non-vanishing coupling of the trace $h$ to the trace of $T_{\mu \nu}$. We will shortly outline this procedure.

Consider the FP theory of a massive spin 2 field $h_{\mu \nu}$ coupled to the energy-momentum tensor $T_{\mu \nu}$

$$
\mathscr{L}_{\mathrm{FP}}=\frac{1}{2}\left\{h^{\mu v} G(h)_{\mu v}^{l i n}-m^{2}\left(h^{\mu v} h_{\mu v}-h^{2}\right)\right\}+h_{\mu v} T^{\mu v} .
$$

Stückelberg fields $V_{\mu}$ and $\phi$ are introduced by the following field redefinition

$$
h_{\mu v}=h_{\mu v}^{\prime}+\frac{1}{m}\left(\partial_{\mu} V_{v}+\partial_{v} V_{\mu}\right)+\frac{2}{m^{2}} \partial_{\mu} \partial_{v} \phi
$$

\footnotetext{
${ }^{2}$ Latin indices denote spatial components of the tensors only. We have explicitly written out the time components.
} 
For generic $m$ the Stückelberg fields are pure gauge as can be seen for example by the gaugetransformations

$$
\delta V_{\mu}=\partial_{\mu} \Xi, \quad \delta \phi=-m \Xi,
$$

and a similar one for $h_{\mu \nu}^{\prime}$ and $V_{\mu}$ with a vector gauge-parameter. These gauge transformations leave the action (2.9) invariant provided that we require that the energy-momentum tensor is conserved, i.e. $\partial^{\mu} T_{\mu \nu}=0$.

Inserting (2.10) into (2.9), together with the requirement $\partial^{\mu} T_{\mu v}=0$, we can take the limit $m \rightarrow 0$ and obtain an action where the vector $V_{\mu}$ decouples, but we get off-diagonal terms mixing $\phi$ and $h_{\mu \nu}^{\prime}$. This action, however, can be diagonalized by shifting $h_{\mu \nu}^{\prime}$ with $\eta_{\mu \nu} \phi$, at the cost of introducing the infamous coupling of $\phi$ to the trace of the energy-momentum tensor $\eta^{\mu v} T_{\mu v}$.

However, it has been argued by Vainshtein that the linear theory is inaccurate if we take the mass to zero [4] (see also [5] and the recent review [6]). He showed that the solutions of the linearized FP theory are only valid far outside a characteristic distance scale, the Vainshtein radius

$$
R_{V}=\left[M /\left(m^{4} M_{P}^{2}\right)\right]^{1 / 5}
$$

Here, $M_{P}$ is the Planck mass and $M$ the mass of a (heavy) central object, e.g. the sun, that determines the metric that our 'test' mass $m$ probes. The Vainshtein radius goes to infinity when the mass of the graviton $m$ is sent to zero. At distances smaller than $R_{V}$ non-linearities begin to dominate and the linear approximation cannot describe the massless limit.

For distances $r \gg R_{V}$ the Stückelberg scalar field $\phi$ behaves with the usual $1 / r$ Coulomb form causing an extra attractive scalar force. However, for distances $r \ll R_{V}$ there is an extra repulsive force that cancels the scalar force responsible for the vDVZ discontinuity, and GR is restored inside the Vainshtein radius.

To find a non-linear generalization of the FP action, that does not lead to ghosts and yields General Relativity in the limit $m \rightarrow 0$, is a highly non-trivial task. In the following section we introduce two recently proposed actions whose linearized versions reduce to (2.2).

\section{New Massive Gravity}

One possibility to acquire a theory with massive spin-2 modes, apart from explicit mass terms, is to introduce higher-derivative terms in the action. Such higher-order derivative modifications of gravity where considered in [7] in an effort to improve the renormalizability properties of general relativity.

The main drawback of higher-derivative theories is that these models in general suffer from ghost instabilities, because of the higher-order time derivatives in their actions. One way to circumvent this conclusion is to confine oneself to gravity in three space-time dimensions. In three dimensions the 'massless' Einstein modes do not propagate. Thus, any potentially ghost-like feature connected to them would be harmless, since it does not constitute any physical degree of freedom. 
Using this idea we can write down a higher-derivative action with a 'healthy' massive spin-2 mode and a massless spin-2 ghost mode, which is pure gauge. This can be seen by writing the linearized action with the help of an auxiliary field, see eqs. (3.6) below.

To be specific, we consider the three-dimensional higher-derivative action of New Massive Gravity (NMG) [8]

$$
S_{\mathrm{NMG}}=\frac{1}{\kappa^{2}} \int \mathrm{d}^{3} x \sqrt{-g}\left\{R+\frac{1}{m^{2}}\left(R^{\mu v} R_{\mu v}-\frac{3}{8} R^{2}\right)\right\} .
$$

The linearized field equations around a Minkowski vacuum are found to be

$$
\left(\square-m^{2}\right) G_{\mu \nu}^{\operatorname{lin}}=0, \quad R^{\text {lin }}=0,
$$

where $G_{\mu \nu}^{\mathrm{lin}}$ and $R^{\mathrm{lin}}$ are the linearized Einstein tensor and Ricci scalar, respectively. Writing the linearized Einstein tensor in the form of eq. (2.3) we note that the equations (3.2) can be interpreted as a "boosted-up" version of the following FP equations for a symmetric perturbation $H_{\mu \nu}$ :

$$
\left(\square-m^{2}\right) H_{\mu v}=0, \quad \partial^{\mu} H_{\mu v}=H=0 .
$$

By identifying $H_{\mu \nu}=G_{\mu \nu}^{\mathrm{lin}}(h)$ we solve for the differential subsidiary constraint, while the tracelessness of $H_{\mu \nu}$ is encoded in the vanishing of $R^{\mathrm{lin}}$. Thus, we get the FP equations for a massive spin-2 field from NMG.

It is instructive to see how, using auxiliary fields, we can make manifest the connection of NMG to the FP theory, at the level of the Lagrangian. Using a symmetric auxiliary field $f_{\mu \nu}$ we can rewrite the action (3.1) as

$$
S_{\text {aux }-\mathrm{NMG}}=\frac{1}{\kappa^{2}} \int \mathrm{d}^{3} x \sqrt{-g}\left\{R+f^{\mu v} G_{\mu v}-\frac{m^{2}}{4}\left(f^{\mu v} f_{\mu v}-f^{2}\right)\right\} .
$$

The linearized Lagrangian will take the form

$$
\mathscr{L}_{\text {lin-aux-NMG }}=\left(\hat{f}^{\mu v}-\frac{1}{2} h^{\mu v}\right) G_{\mu v}^{\operatorname{lin}}(h)-\frac{m^{2}}{4}\left(\hat{f}^{\mu v} \hat{f}_{\mu v}-\hat{f}^{2}\right),
$$

where $\hat{f}_{\mu v}$ denotes the perturbation of $f_{\mu \nu}$. Next, we can recombine the terms of (3.5) to obtain

$$
\mathscr{L}_{\mathrm{FP}-\mathrm{NMG}}=-\frac{1}{2} \tilde{h}^{\mu v} G_{\mu v}^{\operatorname{lin}}(\tilde{h})+\frac{1}{2}\left[\hat{f}^{\mu v} G_{\mu v}^{\operatorname{lin}}(\hat{f})-\frac{m^{2}}{2}\left(\hat{f}^{\mu v} \hat{f}_{\mu v}-\hat{f}^{2}\right)\right],
$$

where we have defined $\tilde{h}_{\mu v}=h_{\mu v}-\hat{f}_{\mu v}$. In this form we can clearly identify the non-propagating Einstein mode, $\tilde{h}_{\mu v}$, as well as its ghost-like nature, and the decoupled, ghost-free, massive spin-2 mode, $\hat{f}_{\mu \nu}$, given by a FP Lagrangian.

The possibility of having massive spin-2 excitations in three dimensions was realized earlier in [9]. This theory does not reduce to the FP theory at the linearized level. This is due to the fact that it is not parity invariant and propagates only one of the two helicity states of the massive graviton in NMG. To be more specific, the combination of two topological terms, the Einstein-Hilbert action 
plus a Chern-Simons term, leads to so-called topologically massive gravity (TMG). This is a ghostfree and parity-odd theory that propagates one massive mode of helicity either +2 or -2 , depending on the sign of the mass parameter $\mu$. The action with cosmological constant $\Lambda$ is given by

$$
S_{\mathrm{TMG}}=\int \mathrm{d}^{3} x \sqrt{-g}\left\{R-2 \Lambda+\frac{1}{2 \mu} \varepsilon^{\lambda \mu v} \Gamma_{\lambda \sigma}^{\rho}\left(\partial_{\mu} \Gamma_{\rho v}^{\sigma}+\frac{2}{3} \Gamma_{\mu \tau}^{\sigma} \Gamma_{v \rho}^{\tau}\right)\right\}
$$

TMG is in some sense the 'square-root' of NMG because it contains half as many degrees of freedom. Also its field equations can be understood as the square-root of the FP equations. We will not consider this model further in this note.

It is known that higher-derivative theories allow for cosmological solutions even without including a cosmological constant term in the action [10]. We can nevertheless define a 'cosmological' version of NMG, i.e. the action (3.1) supplemented by a cosmological parameter. The cosmological constant is then a function of this parameter and other (mass) parameters in the action. The mass $M^{2}$ of the propagating mode in cosmological NMG is given by a combination of the parameter $m^{2}$ that multiplies the fourth order term and the cosmological parameter. In the limit $M^{2} \rightarrow 0$ the massive mode becomes massless and degenerates with the Einstein mode. Such a degeneration of solutions leads to so-called logarithmic or critical gravities [11], which are holographically dual to logarithmic conformal field theories, see e.g. [12].

In conclusion, NMG is an interesting way to describe massive gravity in three dimensions. The two massive graviton modes of helicity +2 and -2 are the only modes that propagate in the bulk. The massless excitations are pure gauge. This, however, is not so in higher dimensions. Therefore, the main drawback of NMG is that it serves as a unitary model of massive gravity only in three dimensions. In the following we will discuss a different model that describes massive gravity also in higher dimensions.

\section{Massive Gravity}

To construct non-linear generalizations of the FP mass term without higher-derivative 'interaction' terms an additional non-dynamical reference metric $f_{\mu \nu}$ is required. Recently, a class of ghost-free theories have been proposed by de Rham, Gabadadze and Tolley (dRGT) [13]. ${ }^{3}$ The interaction is given by a matrix of the form $\sqrt{g^{-1} f}$, where the square root of the matrix is defined such that $\sqrt{g^{-1} f} \sqrt{g^{-1} f}=g^{\mu \lambda} f_{\lambda v}$. Their original analysis was done for a flat background $f_{\mu \nu}=\eta_{\mu \nu}$. In $d$ dimensions the dRGT action can be written in the following form

$$
S_{\mathrm{dRGT}}=\frac{M_{P}^{d-2}}{2} \int \mathrm{d}^{d} x \sqrt{-g}\left\{R-\frac{m^{2}}{4} \sum_{n=0}^{d} \beta_{n} S_{n}\left(\sqrt{g^{-1} \eta}\right)\right\} .
$$

The first term inside the brackets is the Einstein-Hilbert kinetic term for the metric $g_{\mu v}$, the second one corresponds to a potential term containing no derivatives of the dynamical metric $g_{\mu \nu}$ but depending explicitly on $\eta_{\mu \nu}$. The Einstein-Hilbert part of this action is invariant under diffeomorphisms but the mass term breaks this symmetry due to the appearance of the fixed background

\footnotetext{
${ }^{3}$ Earlier work on massive gravity and bi-metric gravity goes back to $[14,15,16,17]$.
} 
metric $\eta_{\mu v}$. The coefficients $\beta_{n}$ are free parameters and the $n$-th elementary symmetric polynomial of the eigenvalues of the matrix square root of $g^{\mu \rho} \eta_{\rho v}$ is represented by $S_{n}$.

This potential term has $d+1$ parameters, but one can discard two of them taking into account that the first and the $d$-th symmetric polynomials are just cosmological constants for $g_{\mu \nu}$ (and $\eta_{\mu \nu}$, for non-flat $\eta_{\mu v}$ ). Another coefficient can be eliminated by taking $m$ to be the mass of the massive spin-2 mode and set

$$
-d ! \sum_{k=0}^{d} \frac{\beta_{k}}{k !(d-k) !}+(d-2) ! \sum_{k=2}^{d} \frac{\beta_{k}}{(k-2) !(d-k) !}=-8 .
$$

Therefore, in addition to the mass and the cosmological constants, the theory has $d-2$ free parameters.

It has been shown [18] that the dRGT massive gravity model in $d$ dimensions has a dynamically equivalent Vielbein formulation, by introducing the Vielbein fields $E_{\mu}^{A}(x)$

$$
g_{\mu v}=E_{\mu}^{A} E_{v}{ }^{B} \eta_{A B} .
$$

The action now reads

$$
\begin{aligned}
S_{\mathrm{dRGT}}=\frac{M_{P}{ }^{d-2}}{2}( & \left(\int \mathrm{d}^{d} x \operatorname{det}(E) R[E]\right. \\
& \left.-\frac{m^{2}}{4} \int \sum_{n=0}^{d} \frac{\beta_{n}}{n !(d-n) !} \tilde{\varepsilon}_{A_{1} A_{2} \ldots A_{d}} \mathbf{1}^{A_{1}} \wedge \ldots \wedge \mathbf{1}^{A_{n}} \wedge \mathbf{E}^{A_{n+1}} \wedge \ldots \wedge \mathbf{E}^{A_{d}}\right),
\end{aligned}
$$

where the first term is the Einstein-Hilbert action in Vielbein form, and the potential terms are written using wedge products of the Vielbein one-forms $\mathbf{E}^{A}=E_{\mu}{ }^{A} d x^{\mu}$ and unit one-forms which can be thought of as Vielbeins for the flat background metric $\mathbf{1}^{A}=\delta_{\mu}{ }^{A} d x^{\mu}$.

The advantage of using this Vielbein formulation is that instead of working with the symmetric polynomials and matrix square roots, it contains wedge products of the possible combinations of the Vielbeins which is much simpler. In this form, it is also easier to show that the Boulware-Deser ghost is absent to all orders in the fields and beyond any decoupling limits.

It is worth pointing out that the massless limit of dRGT gravity must yield Einstein gravity. Unlike the higher-derivative theories discussed earlier, dRGT models, in any dimension, propagate only the massive spin-2 mode. Taking the mass to zero must lead to a theory with only a massless spin- 2 mode, inevitably Einstein gravity. The usual coupling of the scalar to the trace of the energymomentum tensor that plagues the linearized theory vanishes if the value of the graviton mass goes to zero. ${ }^{4}$

Despite successful applications of the dRGT model in cosmological setups much still has to be better understood about these kinds of massive gravity models. For example, for particular potentials the massive gravity model allows for closed timelike curves and therefore is acausal [19]. Additionally, a study of perturbations on top of black hole solutions [20] in the dRGT model and its bi-gravity extension (see section 5) reveals that at the linear level the bi-Schwarzschild black hole solutions are unstable. These results may indicate that static black holes in massive gravity do not exist.

\footnotetext{
${ }^{4}$ See, e.g., [5] for a general discussion.
} 


\section{Generalizations}

In this section we would like to discuss generalizations and further developments connected to the two theories of massive gravity introduced in sections 3 and 4.In this section we restrict to three spacetime dimensions.

\subsection{Bi-metric gravity}

We first consider a particular class of bi-metric theories—-thus, two dynamical spin-2 fields—and show how, by taking special limits, it leads to the three theories discussed so far: FP, NMG and three-dimensional dRGT massive gravity [21]. In particular, we consider the bi-metric theory of [22].

We start by discussing the model. It will be convenient to write the actions in first-order formalism. Using the Vielbein formulation of [18] the Lagrangian takes the form ${ }^{5}$

$$
\begin{aligned}
\mathscr{L}_{\mathrm{bi}-\text { grav }}= & -\sigma M_{1} e_{1 a}\left(\mathrm{~d} \omega_{1}^{a}+\frac{1}{2} \varepsilon_{b c}^{a} \omega_{1}^{b} \omega_{1}^{c}\right)-M_{2} e_{2 a}\left(\mathrm{~d} \omega_{2}^{a}+\frac{1}{2} \varepsilon_{b c}^{a} \omega_{2}^{b} \omega_{2}^{c}\right) \\
& -\frac{1}{4} M_{12} m^{2} \varepsilon_{a b c}\left(e_{1}+e_{2}\right)^{a}\left(e_{1}-e_{2}\right)^{b}\left(e_{1}-e_{2}\right)^{c} \\
& +\alpha M_{12} m^{2} \varepsilon_{a b c}\left(e_{1}-e_{2}\right)^{a}\left(e_{1}-e_{2}\right)^{b}\left(e_{1}-e_{2}\right)^{c} .
\end{aligned}
$$

There are two Dreibeins $e_{I \mu^{a}}{ }^{a}$ and corresponding spin-connections $\omega_{I \mu}{ }^{a}$, with $I=1,2$. Products of the one-forms $e_{I \mu}{ }^{a}$ and $\omega_{I \mu}{ }^{a}$ are understood as exterior products. The three mass parameters $M_{1}, M_{2}, m$ and the two dimensionless parameters $\sigma$ and $\alpha$, where $\sigma$ takes only the values \pm 1 , determine $M_{12}=\sigma M_{1} M_{2} /\left(\sigma M_{1}+M_{2}\right)$ and complete the action.

In the following we describe the linearized theory and show how to obtain NMG and dRGT gravity, in Dreibein formalism, from the action (5.1) in certain parameter limits.

\section{The Fierz-Pauli model}

We first show how we can obtain the FP theory by linearizing the model above. We linearize around a flat background and, for simplicitly, take $\sigma=1$ and set $M_{1}=M_{2}=M$. The fluctuations are $h_{I \mu}{ }^{a}$ and $v_{I \mu}{ }^{a}$, i.e.

$$
e_{I \mu}{ }^{a}=\delta_{\mu}{ }^{a}+\frac{1}{\sqrt{M}} h_{I \mu}{ }^{a}, \quad \omega_{I \mu}^{a}=\delta_{\mu}^{a}+\frac{1}{\sqrt{M}} v_{I \mu}{ }^{a},
$$

with $\delta_{\mu}{ }^{a} \delta_{v}{ }^{b} \eta_{a b}=\eta_{\mu \nu}$. With these simplifications the Lagrangian (5.1), after taking the limit $M \rightarrow \infty$, can be diagonalized in terms of new variables $h_{ \pm \mu}^{a}=h_{1 \mu^{a}} \pm h_{2 \mu}{ }^{a}$ and $v_{ \pm \mu}{ }^{a}=v_{1 \mu}{ }^{a} \pm v_{2 \mu}{ }^{a}$ :

$$
\begin{aligned}
\mathscr{L}_{\text {lin }}= & -\frac{1}{2}\left(h_{+a} \mathrm{~d} v_{+}^{a}+\frac{1}{2} \varepsilon_{a b c} \delta^{a} v_{+}^{b} v_{+}^{c}\right) \\
& -\frac{1}{2}\left(h_{-a} \mathrm{~d} v_{-}^{a}+\frac{1}{2} \varepsilon_{a b c} \delta^{a} v_{-}^{b} v_{-}^{c}+\frac{1}{2} m^{2} \varepsilon_{a b c} \delta^{a} h_{-}^{b} h_{-}^{c}\right) .
\end{aligned}
$$

The first line is linearized Einstein gravity and the second line is the FP Lagrangian (2.2), both in first-order form.

\footnotetext{
${ }^{5}$ This formula and the discussion below was obtained from discussions with Sjoerd de Haan, Wout Merbis and Jan Rosseel.
} 


\section{The NMG limit}

The NMG model has only one dynamical metric, hence we have to fix one Dreibein and spinconnection in terms of the other ones. This can be achieved by setting $\sigma=-1$, substituting

$$
e_{2 \mu^{a}}=e_{1 \mu}{ }^{a}+\frac{\lambda}{m^{2}} f_{\mu}{ }^{a}, \quad \omega_{2 \mu}{ }^{a}=\omega_{1 \mu^{a}}-\lambda h_{\mu}{ }^{a},
$$

and taking the following limit [23]:

$$
\lambda \rightarrow 0, \quad M_{1}, M_{2} \rightarrow \infty, \quad \text { with } \quad M_{1}-M_{2}=\lambda M_{2}=\text { constant }=M_{P} .
$$

This leads to the Lagrangian

$$
\mathscr{L}_{\mathrm{NMG}}=M_{P}\left\{e_{a} R^{a}+h_{a} T^{a}-\frac{1}{m^{2}}\left(f_{a} R^{a}+\frac{1}{2} \varepsilon_{a b c} e^{a} f^{b} f^{c}\right)\right\} .
$$

The curvature and torsion two-forms $R^{a}$ and $T^{a}$ are determined by the dreibein $e_{\mu}{ }^{a}$. The Lagrangian (5.6) is identified with NMG in the Chern-Simons-like formulation of [24]. By solving the equations for the auxiliary field $f_{\mu}{ }^{a}$, substituting this solution back into the action and going to second-order formalism we can go from (5.6) to the Lagrangian given in (3.1).

\section{The dRGT limit}

The three-dimensional dRGT model can be obtained from the Lagrangian (5.1) by choosing $\sigma=$ +1 and setting

$$
e_{2 \mu}{ }^{a}=\delta_{\mu}{ }^{a}+M_{2}^{-1 / 2} \delta e_{2 \mu}{ }^{a} .
$$

If we now take the limit $M_{2} \rightarrow \infty$ the fluctuations $\delta e_{2} \mu^{a}$ decouple from the massive mode $e_{1} \mu^{a}$ and the second metric is fixed to its background value, which we choose to be the flat metric. In this limit $M_{12}=M_{1}=M_{P}$, and the Lagrangian (5.1) becomes

$$
\begin{aligned}
\mathscr{L}_{\mathrm{dRGT}}=M_{P}\{ & -e_{a} R^{a}-\frac{m^{2}}{4} \varepsilon_{a b c}(e+\delta)^{a}(e-\delta)^{b}(e-\delta)^{c} \\
& \left.+\alpha m^{2} \varepsilon_{a b c}(e-\delta)^{a}(e-\delta)^{b}(e-\delta)^{c}\right\} .
\end{aligned}
$$

This is the first-order formulation of dRGT gravity in three dimensions.

We end this section with a short note on the main differences between higher-derivative and dRGT massive gravities. Surprisingly, in three dimensions the a priori very different Lagrangians (5.6) and (5.8) effectively describe the same bulk degrees of freedom: two degrees of freedom which are the two helicity states of a massive graviton in three-dimensional space-time. It is only when one goes to higher dimensions that these two Lagrangians describe rather different theories. A higher-derivative theory analogue to NMG will describe a massless spin-2 plus a massive spin-2, one of them necessarily being a ghost. For a recent effort to create "healthy" higher-dimensional analogues of NMG, see [25]. On the other hand, the dRGT model in higher dimensions still propagates only the massive spin-2 mode. Interestingly, it has been claimed that the acausalities that could be present in dRGT massive gravity are not existent in bi-gravity, but rather are an artefact of freezing out the degrees of freedom of one of the metrics [26]. 


\subsection{Supersymmetry}

One can also consider a supersymmetric generalization of the theories discussed so far. For NMG this has been done in [27]. This was done using only the metric and higher-derivative action. Alternatively, one may consider the auxiliary field version of NMG. For simplicity, we only consider the linearized theory.

For a supersymmetric extension, one needs to obtain the linearized massive spin- 2 multiplet in three dimensions. This multiplet can be obtained by a Kaluza-Klein reduction of the massless multiplet in four dimensions and a truncation to the first massive mode sector. This truncation is consistent only at the linearized level, therefore we obtain only the linearized multiplet in three dimensions. We will give a brief outline on how this is done. We refer to [28] for more details.

We start from the off-shell, four-dimensional, $\mathscr{N}=1$ massless spin-2 multiplet which consists of a symmetric tensor $\hat{h}_{\hat{\mu} \hat{v}}$, a gravitino $\hat{\psi}_{\hat{\mu}}$, an auxiliary vector $\hat{A}_{\hat{\mu}}$ and two auxiliary scalars $\hat{M}$ and $\hat{N}$ (all hatted fields are four-dimensional and unhatted fields are three-dimensional fields). The supersymmetry rules, with constant spinor parameter $\varepsilon$, and gauge transformations of these fields, with local vector parameter $\hat{\Lambda}_{\hat{\mu}}$ and local spinor parameter $\hat{\eta}$, are given by [29, 30]:

$$
\begin{aligned}
\delta \hat{h}_{\hat{\mu} \hat{v}} & =\bar{\varepsilon} \Gamma_{(\hat{\mu}} \hat{\psi}_{\hat{v})}+\partial_{(\hat{\mu}} \hat{\Lambda}_{\hat{v})}, \\
\delta \hat{\psi}_{\hat{\mu}} & =-\frac{1}{4} \Gamma^{\hat{\rho} \hat{\lambda}} \partial_{\hat{\rho}} \hat{h}_{\hat{\lambda} \hat{\mu}} \varepsilon-\frac{1}{12} \Gamma_{\hat{\mu}}\left(\hat{M}+i \Gamma_{5} \hat{N}\right) \varepsilon+\frac{1}{4} i \hat{A}_{\hat{\mu}} \Gamma_{5} \varepsilon-\frac{1}{12} i \Gamma_{\hat{\mu}} \Gamma^{\hat{\rho}} \hat{A}_{\hat{\rho}} \Gamma_{5} \varepsilon+\partial_{\hat{\mu}} \hat{\eta}, \\
\delta \hat{M} & =-\bar{\varepsilon} \Gamma^{\hat{\rho} \hat{\lambda}} \partial_{\hat{\rho}} \hat{\psi}_{\hat{\lambda}}, \\
\delta \hat{N} & =-i \bar{\varepsilon} \Gamma_{5} \Gamma^{\hat{\rho} \hat{\lambda}} \partial_{\hat{\rho}} \hat{\psi}_{\hat{\lambda}}, \\
\delta \hat{A}_{\hat{\mu}} & =\frac{3}{2} i \bar{\varepsilon} \Gamma_{5} \Gamma_{\hat{\mu}}^{\hat{\rho} \hat{\lambda}} \partial_{\hat{\rho}} \hat{\psi}_{\hat{\lambda}}-i \bar{\varepsilon} \Gamma_{5} \Gamma_{\hat{\mu}} \Gamma^{\hat{\rho} \hat{\lambda}} \partial_{\hat{\rho}} \hat{\psi}_{\hat{\lambda}} .
\end{aligned}
$$

Next, we split the four-dimensional coordinates as $x^{\hat{\mu}}=\left(x^{\mu}, x^{3}\right)$, write all fields as a Fourier series and impose reality conditions on the bosonic fields and Majorana conditions on the fermionic fields. After this, we project onto the lowest, massive (!), Kaluza-Klein sector ( $n=1$ modes) and obtain the transformation rules of all the fields. Since this procedure effectively doubles our variables we have to truncate half of the fields and the gauge parameters thereby getting rid of the so-called central charge transformations.

This way we find a three-dimensional massive multiplet whose supersymmetry algebra closes off-shell. Formally, this multiplet contains the same fields that occur in a massless mutiplet with $\mathscr{N}=2$ supersymmetry. However, in the massive case the gauge-symmetries of the four-dimensional fields carry over to the three-dimensional fields in such a way that some of them are subject to trivial shift-symmetries. Hence these fields are not physical but rather represent Stückelberg symmetries and can be gauged away.

We fix all Stückelberg symmetries by choosing a certain gauge, taking into account the compensating gauge transformations. Thus we obtain the final form of the supersymmetry rules of the three-dimensional $\mathscr{N}=1$ off-shell massive spin-2 multiplet. The off-shell supersymmetric version 
of FP is then given by the action

$$
\begin{aligned}
I_{m \neq 0}=\int \mathrm{d}^{3} x\{ & h^{\mu v} G_{\mu \nu}^{\operatorname{lin}}(h)-m^{2}\left(h^{\mu v} h_{\mu v}-h^{2}\right) \\
& -4 \bar{\psi}_{\mu} \gamma^{\mu \nu \rho} \partial_{\nu} \psi_{\rho}-4 \bar{\chi}_{\mu} \gamma^{\mu \nu \rho} \partial_{\nu} \chi_{\rho}+8 m \bar{\psi}_{\mu} \gamma^{\mu v} \chi_{v} \\
& \left.-\frac{2}{3} M^{2}-\frac{2}{3} N^{2}+\frac{2}{3} P^{2}+\frac{2}{3} A_{\mu} A^{\mu}\right\} .
\end{aligned}
$$

The first line is the standard FP action. The fermionic off-diagonal mass term can be diagonalized by going to a basis in terms of the sum and difference of the two vector-spinors $\psi_{\mu}$ and $\chi_{\mu}$ each of which describes one helicity state with helicity either $+3 / 2$ or $-3 / 2$. The "trivial" auxiliary fields $M, N, P$ and $A_{\mu}$ are needed for off-shell closure of the supersymmetry algebra. The algebra is determined in terms of the supersymmetry transformation rules

$$
\begin{aligned}
\delta h_{\mu v} & =\bar{\varepsilon} \gamma_{(\mu} \psi_{v)}+\frac{1}{m} \bar{\varepsilon} \partial_{(\mu} \chi_{v)}, \\
\delta \psi_{\mu} & =-\frac{1}{4} \gamma^{\rho \lambda} \partial_{\rho} h_{\lambda \mu} \varepsilon+\frac{1}{12} \gamma_{\mu}(M+P) \varepsilon+\frac{1}{12 m} \partial_{\mu}\left(N+\gamma^{\rho} A_{\rho}\right) \varepsilon \\
\delta \chi_{\mu} & =\frac{1}{4} m \gamma^{\rho} h_{\rho \mu} \varepsilon+\frac{1}{4} A_{\mu} \varepsilon-\frac{1}{12} \gamma_{\mu}\left(N+\gamma^{\rho} A_{\rho}\right) \varepsilon-\frac{1}{12 m} \partial_{\mu}(M-2 P) \varepsilon \\
\delta M & =\bar{\varepsilon} \gamma^{\rho \lambda} \partial_{\rho} \psi_{\lambda}-m \bar{\varepsilon} \gamma^{\rho} \chi_{\rho} \\
\delta N & =-\bar{\varepsilon} \gamma^{\rho \lambda} \partial_{\rho} \chi_{\lambda}+m \bar{\varepsilon} \gamma^{\rho} \psi_{\rho} \\
\delta P & =\frac{1}{2} \bar{\varepsilon} \gamma^{\rho \lambda} \partial_{\rho} \psi_{\lambda}+m \bar{\varepsilon} \gamma^{\rho} \chi_{\rho}, \\
\delta A_{\mu} & =\frac{3}{2} \bar{\varepsilon} \gamma_{\mu}^{\rho \lambda} \partial_{\rho} \chi_{\lambda}-\bar{\varepsilon} \gamma_{\mu} \gamma^{\rho \lambda} \partial_{\rho} \chi_{\lambda}-\frac{1}{2} m \bar{\varepsilon} \gamma_{\mu}{ }^{\rho} \psi_{\rho}+m \bar{\varepsilon} \psi_{\mu},
\end{aligned}
$$

which also leave invariant the action (5.10).

The off-shell massive spin-2 multiplet (5.11), together with the off-shell massless spin-2 multiplet given in [27], can be used to write a supersymmetric version of linearized NMG in the auxiliary field form. This can be achieved by separately supersymmetrizing the massless and massive parts of the action (3.6) using the known massless off-shell multiplet and the given massive off-shell multiplet (5.11), respectively.

A non-linear version of the multiplet (5.11) is not known and a supersymmetric auxiliary field version of (non-linear) NMG does not (yet) exist. To obtain such a non-linear extension most likely a superspace approach is needed.

\section{Outlook}

We have motivated and discussed recent efforts towards a consistent theory of massive gravity. After reviewing the work of Fierz and Pauli on a linearized theory of massive spin-2 particles we focused on two recently proposed models of massive gravity, NMG and dRGT gravity. We discussed the pros and cons of these two models and shortly discussed different generalizations. 
One extension we discussed, in the case of three-dimensions, was the incorporation of the different models into a bi-gravity theory. Bi-gravity theories have received a lot of attention recently. They allow for interesting cosmological solutions. From a more theoretical point of view one can ask questions such as how the intrinsic "good" features of these models, like ghost-freedom, vanish in certain limits [26].

A second generalization we discussed concerns supersymmetric extensions. Three-dimensional supergravity theories with higher-curvature actions have been studied in [27]. An interesting open question is whether one can combine the two extensions we discussed and construct a supersymmetric bi-gravity theory. Such and other questions make it clear that much more work needs and can to be done in the field of massive gravity.

\section{Acknowledgements}

EB would like to thank the organizers of the XVIII European Workshop on String Theory for the opportunity to present a talk and the organizers of the Corfu Summer Institute 2012 for providing a stimulating environment.

\section{References}

[1] T. Clifton, P. G. Ferreira, A. Padilla and C. Skordis, "Modified gravity and cosmology," Phys.Rept. 531 (2012) 1-189.

[2] K. Hinterbichler, "Theoretical Aspects of Massive Gravity," Phys.Mod.Phys. 84 (2012) 671-710.

[3] H. van Dam and M. J. G. Veltman, "Massive and massless Yang-Mills and gravitational fields," Nucl.Phys. B22 (1970) 397-411.

V. Zakharov, "Linearized gravitation theory and the graviton mass," JETP Lett. 12 (1970) 312.

[4] A. I. Vainshtein, "To the problem of nonvanishing gravitation mass," Phys.Lett. B39 (1972) 393-394.

[5] C. Deffayet, G.R. Dvali, G. Gabadadze and A. I. Vainshtein, "Nonperturbative continuity in graviton mass versus perturbative discontinuity," Phys.Rev. D65 (2002) 044026.

[6] E. Babichev and C. Deffayet, "An introduction to the Vainshtein mechanism," arXiv:1304.7240.

[7] K. S. Stelle, "Renormalization of Higher Derivative Quantum Gravity," Phys.Rev. D16 (1977) 953.

[8] E. A. Bergshoeff, O. Hohm and P. K. Townsend, "Massive gravity in three dimensions," Phys.Rev.Lett. 102 (2009) 201301.

[9] S. Deser, R. Jackiw and S. Templeton, "Three-Dimensional Massive Gauge Theories," Phys.Rev.Lett. 48 (1982) 975-978.

[10] D. G. Boulware and S. Deser, "String Generated Gravity Models," Phys.Rev.Lett. 55 (1985) 2656.

[11] D. Grumiller and N. Johansson, "Instability in cosmological topologically massive gravity at the chiral point," JHEP 0807 (2008) 134.

[12] E. A. Bergshoeff, S. de Haan, W. Merbis, J. Rosseel and T. Zojer, "Three-dimensional tricritical gravity," Phys.Rev. D86 (2012) 064037.

D. Grumiller, W. Riedler, J. Rosseel and T. Zojer, "Holographic applications of logarithmic conformal field theories," arXiv:1302.0280. 
[13] C. de Rahm, G. Gabadadze and A. J. Tolley, "Resummation of massive gravity," Phys.Rev.Lett. 106 (2011) 231101.

[14] A. Salam and J. A. Strathdee, "Nonlinear realizations. 1: The Role of Goldstone bosons," Phys.Rev. 184 (1969) 1750.

[15] A. Salam and J. A. Strathdee, "Nonlinear realizations. 2. Conformal symmetry," Phys.Rev. 184 (1969) 1760.

[16] C. J. Isham, A. Salam and J. A. Strathdee, "Spontaneous breakdown of conformal symmetry," Phys.Lett. B31 (1970) 300.

[17] B. Zumino, "Effective Lagrangians and broken symmetries," in Brandeis University Lectures On Elementary Particles and Quantum Field Theory, (MIT, Cambridge, MA, 1970), Vol. 2, 437-500.

[18] K. Hinterbichler and R. A. Rosen, “Interacting Spin-2 Fields,” JHEP 1207 (2012) 047.

[19] S. Deser and A. Waldron, "Acausality of massive gravity," Phys.Rev.Lett. 110 (2013) 111101.

[20] E. Babichev and A. Fabbri, "Instability of black holes in massive gravity," arXiv:1304.5992.

[21] C. de Rham, G. Gabadadze, D. Pirtskhalava, A. J. Tolley and I. Yavin, "Nonlinear Dynamics of 3D Massive Gravity,” JHEP 1106 (2011) 028 [arXiv:1103.1351 [hep-th]].

[22] S. F. Hassan and R. A. Rosen, "Bimetric Gravity from Ghost-free Massive Gravity," JHEP 1202 (2012) 127.

[23] M. F. Paulos and A. J. Tolley, "Massive Gravity Theories and limits of Ghost-free Bigravity models," JHEP 1209 (2012) 002.

[24] O. Hohm, A. Routh, P. K. Townsend and B. Zhang, "On the Hamiltonian form of 3D massive gravity," Phys.Rev. D86 (2012) 084035.

[25] E. A. Bergshoeff, J. J. Fernandez-Melgarejo, J. Rosseel and P. K. Townsend, “'On 'New Massive' 4D Gravity," JHEP 1204 (2012) 070.

[26] S. F. Hassan, A. Schmidt-May and M. von Strauss, "Higher Derivative Gravity and Conformal Gravity From Bimetric and Partially Massless Bimetric Theory," arXiv:1303.6940.

[27] R. Andringa, E. A. Bergshoeff, M. de Roo, O. Hohm, E. Sezgin and P. K. Townsend, "Massive 3D Supergravity," Class.Quant.Grav. 27 (2010) 025010.

[28] E. A. Bergshoeff, M. Kovačević, L. Parra, J. Rosseel, Y. Yin and T. Zojer, "New Massive Supergravity and Auxiliary Fields," arXiv:1304.5445.

[29] Ferrara, S. and van Nieuwenhuizen, P., "The Auxiliary Fields of Supergravity," Phys.Lett. B74 (1978) 333.

[30] Stelle, K.S. and West, Peter C., "Minimal Auxiliary Fields for Supergravity," Phys.Lett. B74 (1978) 330. 